Outcomes Success to date has been extremely positive with outcome measures exceeding expectation across all areas.

Application into Hospice Practice An action plan was developed and implementation commenced of the recommended actions. The new documentation was rolled out on the 30 November and is now being used for all patients who are admitted to the in-patient unit. Care plans were redesigned to encourage planning and documentation of personalised care. Records audit was then done every week, all patients records are audited without exception.

Feedback from issues found through audit is given directly to the staff member involved as soon as possible either face to face or by email and clinical supervision documentation is completed for evidence of feedback. Compliance of feedback is checked the following week whilst doing the weekly audit. The nurse coordinators' role defined to include monitoring of documentation following admission to ensure compliance sustaining improvements. There remained a need to continue weekly audits until staff have adapted and familiarised themselves with the documentation, by March 2017 following evidence of sustained compliance to the audit requirements, the audit was conducted fortnightly for three months and if remains stable will then be downscaled to monthly. Documentation is clearer and evidences patient and family engagement and consent in personalised care planning. Documentation compliance has improved.

\section{P-175 AN AUDIT OF DOCUMENTATION OF MENTAL CAPACITY ASSESSMENTS AT ST JOSEPH'S HOSPICE}

Anna Bradley, Gabrielle Rose, Hayley Sherratt, Margaret Clifford. St Joseph's Hospice, London, UK

\subsection{6/bmispcare-2017-hospice.200}

Introduction Since the introduction of the Mental Capacity Act (MCA) in 2005, there has been much debate what constitutes an adequate MCA assessment. It is important to keep comprehensive documentation of MCA assessments as they can be challenged. Hospices (along with other healthcare services) need to ensure their staff are competent in conducting MCA assessments and that their documentation is clear and consistent.

Aims To evaluate the quality of MCA assessment and documentation for patients under the care of the hospice.

Methods All MCA assessments conducted in a 34-bed hospice over a six -month period were reviewed retrospectively using an audit tool developed by the British Psychological Society.

Results and Recommendations 44 assessments for 20 patients were analysed. Assessments were generally well documented: the rationale was clear (91\%); the decision to be made was clearly stated (95\%) and the diagnostic and functional tests were specifically addressed (91\%). Areas where practice could be improved included: assessing capacity for one decision at a time; documenting any communication difficulties and how these are overcome; clearly documenting recommendations as a result of the assessment; and identifying the need for repeat assessments. As part of a multidisciplinary discussion, amendments have been made to the MCA assessment documentation template. Results have been disseminated and an education session held to increase awareness, and the hospice documentation template updated, prior to re-audit in August 2017. Conclusions MCA assessment documentation was generally clear and appeared to show staff understood the basic principles of the MCA. It is hoped that updating the documentation template, in addition to staff education, will address the areas requiring improvement.

\section{Service development, models and collaborative working}

\section{P-176 I WANT TO DIE ALONE NURSE, I WANT TO DIE AT HOME}

Jane Carpenter. St Helena Hospice, Colchester, UK

10.1136/bmjspcare-2017-hospice.201

Supporting a patient with limited family and financial resource to die alone at home, when some people were profoundly uncomfortable with their end of life wishes proved a difficult, but not insurmountable, challenge.

Background The concept of a 'Good Death' (Ko et al., 2015) is widely accepted, dying alone is seen as an aspect of 'bad death'. The Mental Capacity Act (2005) makes it clear, people have a right to make informed choices; these choices should not be measured against those others might make. Patients who want to die alone are not always able to do so - wellmeaning friends, neighbours and professionals can create barriers to prevent lone death including detailing safety, medicines and vulnerable adult concerns.

Results The patient died at home, alone, what did we do ?

- Enabled the patient to be explicit about their choices, and communicate them to friends, neighbours and professionals effectively

- Recognised that dying alone was a valid choice, made with capacity, and fully in tune with the context of this person's life

- Reflected on our insecurities, helped carers to do the same, recognising that our fear can create a barrier to patient choice

- Brought together the widest multidisciplinary team including social services, carers, neighbours, helping them to understand the patient's viewpoint, right to autonomy and self-determination

- Honestly addressed risks to patient safety, safeguarding, medicines management, access to patient's home - minimising those risks using technology, equipment adaptations and creative problem solving

- Rapid response hospice team involved.

Conclusions The biggest challenge was to overcome preconceptions of what is a good death to enable the patient to have their choices honoured. Creating common understanding with good communication, having a clear plan, and taking a degree of shared risk empowered real patient choice at end of life. An alone death is not necessarily a bad death.

\section{P-177 DISCHARGE BUDDIES - FEELING SAFE GOING HOME}

Judith RM Williams. Martlets Hospice, Hove, UK

10.1136/bmjspcare-2017-hospice.202

Background When patients are discharged from a hospice inpatient unit many report feeling vulnerable and abandoned; 
in response, this hospice has set up a Volunteer Discharge Buddy Service to help alleviate these feelings, to maintain patient safety and restore confidence on returning home.

Aims The service aims to support patients and families with practical tasks during and following discharge. The Buddies offer support on the day of discharge and make follow-up visits for up to five weeks. They liaise with community services and signpost carers and families to other services as needed.

Methods To recruit Buddies, there is a rigorous selection process and intensive two-day training programme, which includes issues around working with palliative care patients; lone working policy; safeguarding and other relevant topics. The Discharge Coordinator allocates patients to Buddies, who meet each other prior to discharge; more complex patients are allocated two Buddies. All visits are recorded in the electronic patient notes. We hold monthly supervision sessions where the Buddies discuss their experiences and bring new ideas to develop the service. We find these invaluable.

Results An evaluation form is sent to each patient. Feedback indicates that patients and families feel very reassured by the service we provide. Feedback from colleagues has also been positive. In response to considerable interest from other hospices, we have developed an information pack to assist them in setting up their own service. Colleagues from other hospices have also visited.

Conclusion Uptake has been excellent and we are planning to recruit more Buddies and extend the service to include a 'sitting' service. Additionally, we have supported the community care teams by relieving them of the simpler issues that patients frequently raise following discharge and prevented possible re-admissions when patients or their families panicked when faced with the reality of being at home.

\section{P-178 CAN WE GIVE DYING HOSPITAL PATIENTS BETTER CARE?}

'Keri Thomas, '1ulie Armstrong Wilson, ${ }^{2}$ Collette Clifford. 'Gold Standards Framework Centre C.I.C. Shrewsbury, UK; ${ }^{2}$ Birmingham University, Birmingham, UK

\subsection{6/bmispcare-2017-hospice.203}

Aims A third of hospital patients are in their last year of life, almost $50 \%$ of people die in hospital, and most do not die where they choose. Poor end of life care in UK hospitals has received much recent attention and is recognised as a key area for improvement. With earlier recognition and a more proactive approach, more might receive better care, be discharged home earlier, with fewer hospital deaths.

The Gold Standards Framework (GSF) is a quality improvement programme delivering proactive person-centred population-based care for people in the last years of life in all settings. We report on the introduction and impact of the first eight years of the GSF Acute Hospitals Programme, with findings from the first GSF Accredited wards.

Method The structured two- year GSF Hospital training programme includes training, tools, support and evaluation at organisational, patient and staff level, including measures of progress and attainment and some independent evaluations. GSF encourages teams to identify patients earlier (aiming for the $30 \%$ figure where appropriate), assess their needs (offering all initial advance care planning discussions) and proactively planning care aligned to preferences, enabling more to die at home.

Results Evaluations indicate significant progress in several key areas in many hospitals and a 'culture change' on the wards. GSF Accredited wards show embedded good practice including earlier identification (average 35\%), more proactive needsbased approach, more offered advance care planning discussions (75\%-95\%), better communication with GPs, reduced hospitalisation and improved more appropriate referrals to palliative care specialists.

Conclusions Findings from the first phases of GSF trained hospitals and the first accredited wards show significant changes, with proactive, better coordinated, person-centred care. A key factor is earlier recognition which is increasingly being attained.

\section{P-179 IMPROVING CARE FOR PATIENTS WHO MAY BE IN THEIR LAST MONTHS OF LIFE: THE LENS OF ACUTE ADMISSIONS}

${ }^{1}$ Susanna Shouls, ${ }^{2}$ Vince Connolly, ${ }^{2}$ Gavin Eyres, ${ }^{1}$ Lesley Goodburn, ${ }^{1}$ Anita Hayes, ${ }^{1}$ Paul Hayes, 'Claire Henry. 'The National Council For Palliative Care, London, UK; ${ }^{2}$ Emergency Care Improvement Programme, NHS Improvement, London, UK

\subsection{6/bmispcare-2017-hospice.204}

Background The aim is to improve the quality of care for patients who may be in their last three months of life who attend or are admitted to hospital in an emergency. Hospitals are an important provider of care for this group of people (Clark et al., 2014). An emergency admission may indicate underlying clinical decline. Quality of experience of care varies (Office for National Statistics., 2015). Acute admission processes are not generally designed to manage care for this group (Bailey et al., 2010). This may result in unwanted inpatient stays and/or treatments (Cardona-Morrell et al., 2016) that affect the person's quality of (their remaining) life. Detail is important: 'sometimes, it's the little things that matter, and that is what you remember' (NHS Improving Quality., 2014). This is in the background of increasing demographic related demand for palliative care (Public Health England., 2015) and associated pressure on services (Lowthian et al., 2010).

Method Four acute hospital Trusts formed a quality improvement collaborative in 2016 with expert clinical, quality improvement and patient experience advice. The methods to diagnose underlying problems and facilitate acute physician engagement included: a 'patient/relative' experience walkthrough, a case file review and analysis of activity data and building on existing plans for improvement/known issues. The Trusts set their own priorities for improvement.

Results The 'walkthrough' highlighted areas for improvement, eg information, signage and mortuary visiting environments. The casefile review facilitated acute physician engagement in three Trusts. These highlighted some excellent practice, which was not consistent. Recognition of dying (69\% - average three Trusts); recognition clinical uncertainty of recovery $(53 \%$ average 3 Trusts); non-beneficial treatment $(65 \%$ average two Trusts). $60 \%$ patients who died in hospital had a prior visit to that Trust (three months) - potential missed opportunity to plan. 